\title{
DETECÇÃO DE CAVIDADES EM ARENITOS UTILIZANDO GRAVIMETRIA, ELETRORRESISTIVIDADE E GPR
}

\author{
João C. Dourado ${ }^{1}$, Walter Malagutti Filho ${ }^{1}$, Antônio C.O. Braga ${ }^{1}$ \& Noedir \\ Nava $^{2}$
}

Received April 25, 2000 / Accepted May 15, 2001

\begin{abstract}
Este trabalho mostra os resultados obtidos com a realização de ensaios geofísicos na em áreas de lavra de areia, no município de Descalvado, SP. O objetivo dos ensaios é o de detectar cavidades no interior de arenitos, pertencentes às Formações Pirambóia e Botucatu. Estas cavidades formaram-se com o rebaixamento do lençol freático, necessário para as operações de extração da areia. As técnicas geofísicas utilizadas foram: gravimetria, eletrorresistividade (caminhamento elétrico dipolo-dipolo) e radar de penetração no solo (GPR). Na primeira etapa dos trabalhos foram utilizados a gravimetria e a eletrorresistividade em uma bancada onde ocorreu um desmoronamento devido ao abatimento do teto de uma cavidade. Os resultados obtidos com estas duas técnicas foram coerentes pois a gravimetria mostrou baixos gravimétricos indicando uma "ausência de massa" na área de influência da cavidade e o caminhamento detectou um esperado aumento de resistividade elétrica nesta mesma área. Na segunda etapa, com a realização dos ensaios de GPR em uma bancada próxima à anterior, foi possível detectar cavidades por meio das feições hiperbólicas detectadas nos radargramas.
\end{abstract}

Palavras-chave: Geofísica aplicada; Gravimetria; Eletrorresistividade; Radar de penetração no solo; Detecção de cavidades.

DETECTION OF CAVITIES IN SANDSTONE USING GRAVITY, RESISTIVITY AND GPR METHODS - This paper shows the results of geophysical research in areas of sand exploration near the city of Descalvado, SP, Brazil. The main objective of this research is to detect cavities inside sandstones belonging to the Piramboia and Botucatu Formations in the Parana sedimentary basin. These cavities were indirectly caused by human activity, i.e., the lowering of the water table for the purpose of sand extraction. The geophysical techniques employed were: gravity, resistivity (dipole-dipole electrical profiling) and ground penetrating radar (GPR). In the first stage of the work gravity and resistivity surveys were used to indicate where exists voids due to the collapse of the roof of a cavity. The results obtained with these two techniques were coherent because the gravity showed a "mass absence" in the area of influence of the cavity and the dipole-dipole electrical profiling detected an increase of electric resistivity in this same area. In the second stage, the GPR survey detected the same cavities by means of the hyperbolic features that appear in the radargrams.

Key words: Applied geophysics; Gravity; Resistivity; Ground penetrating radar; Detection of cavities.

${ }^{1}$ Departamento de Geologia Aplicada - IGCE - Unesp - Campus de Rio Claro

Rio Claro - SP

${ }^{2}$ Mineração Jundu S. A.

Descalvado - SP 


\section{INTRODUÇÃO}

A localização, ou mesmo a simples deteç̧ão de cavidades, sempre se constituiu em um desafio para as técnicas prospectivas à disposição do homem.

A geofísica aplicada, que está experimentando um grande desenvolvimento em função dos avanços ocorridos nas áreas da eletrônica e processamento de dados, de certa maneira, sempre enfrentou este desafio.

Devido às múltiplas feições dos locais onde se localizam as cavidades, sejam elas de origem natural (carsticas, erosivas, etc..) construídas ou provocadas pelo homem (túneis, minas, etc...), não existe ainda uma "receita" que indique quais técnicas geofísicas a utilizar para determinado problema.

Os parâmetros que mais influem em um estudo desta natureza são: o contexto geológico onde se localizam as cavidades, a relação entre seu tamanho e a sua profundidade e o tipo e intensidade do ruído presente na área. Por exemplo, se uma técnica geofísica fornece bons resultados para estudo de um determinado meio geológico em um ambiente rural, pode não fornece-los, quando utilizada no ambiente urbano devido à intensidade de ruídos.

Neste trabalho, mostram-se os resultados dos levantamentos gravimétricos, dos ensaios de resistividade elétrica com a técnica de caminhamento elétrico e de radar de penetração no solo (GPR), realizados na mineração Jundu, no município de Descalvado, SP. Estes ensaios tiveram como objetivo detectar cavidades existentes no interior do arenito, que em alguns locais chegam a causar dificuldades operacionais na extração da areia.

\section{CONTEXTO GEOLÓGICO E A OCORRÊNCIA DAS CAVIDADES}

A mina de Areias Quartzosas da Mineração Jundu S.A., situa-se $5 \mathrm{~km}$ ao sul da cidade de Descalvado - São Paulo, próximo à borda leste da bacia sedimentar do Paraná (Fig. 1).

Segundo Petri \& Fulfaro (1983), a bacia sedimentar do Paraná abrange área de $1.600 .000 \mathrm{~km}^{2}$ no centro-leste da América Latina, dos quais $1.000 .000 \mathrm{~km}^{2}$ situam-se em território brasileiro, englobando parte dos estados de São Paulo, Paraná,
Santa Catarina, Rio Grande do Sul, Mato Grosso e Goiás. Trata-se de uma bacia intracratônica cuja subsidência iniciou-se de forma moderada no Siluriano com apogeu no Devoniano, perdendo intensidade gradativamente até o Cenozóico, com intercalações de movimentos positivos.

A sedimentação foi francamente marinha até o Permiano, a partir de quando passou a ser continental com eventos magmáticos no Cretáceo Inferior.

$\mathrm{Na}$ área em estudo ocorrem as seguintes unidades:

- Formação Pirambóia (Triássico-Jurássico) Arenitos ortoquartzíticos, friáveis, granulometria homogênea média a fina e cores predominantemente claras (branco, amarelo, rosa). Apresentam estratificação cruzada de médio porte tangencial na base e plano paralela sub-horizontal, de deposição fluvial. Estas características são descritas também por Soares (1973);

- Formação Botucatu (Jurássico-Cretáceo) Arenitos dispostos em uma sucessão de estrados intercalados finos, médios e grosseiros, e conglomeráticos na base. A coloração é variada (branca, amarela, rosa, marrom e avermelhada). A estrutura mais freqüente é a estratificação cruzada de médio a pequeno porte, ocorrendo também plano paralela sub-horizontal. Estas características coincidem com a descrição dada à Fácies Torrencial da Fm Botucatu por Soares (1973);

- Intrusões de Diabásio (Cretáceo) - Há indícios da ocorrência de um sill de diabásio alojado nos arenitos da Fm Pirambóia, em cota inferior ao piso da mina, do qual partem alguns diques cortando os arenitos sobrejascentes, acompanhando pequenas falhas verticais existentes, possivelmente originadas pela intrusão do sill;

- Formação St ${ }^{\mathrm{a}}$ Rita do Passa Quatro (Terciário) - Massoli (1981) descreve esta formação como Capeamentos Terciários (?), pouco espessos, mas com ampla distribuição horizontal. Constituem-se de areias em matriz argilosa, sem estruturas sedimentares com cascalheira basal de seixos predominantemente de quartzo. Na área estes sedimentos têm coloração marrom, são muito friáveis e apresentam grânulos de quartzo dispersos em toda a espessura, que é da ordem de 5 a $15 \mathrm{~m}$. 


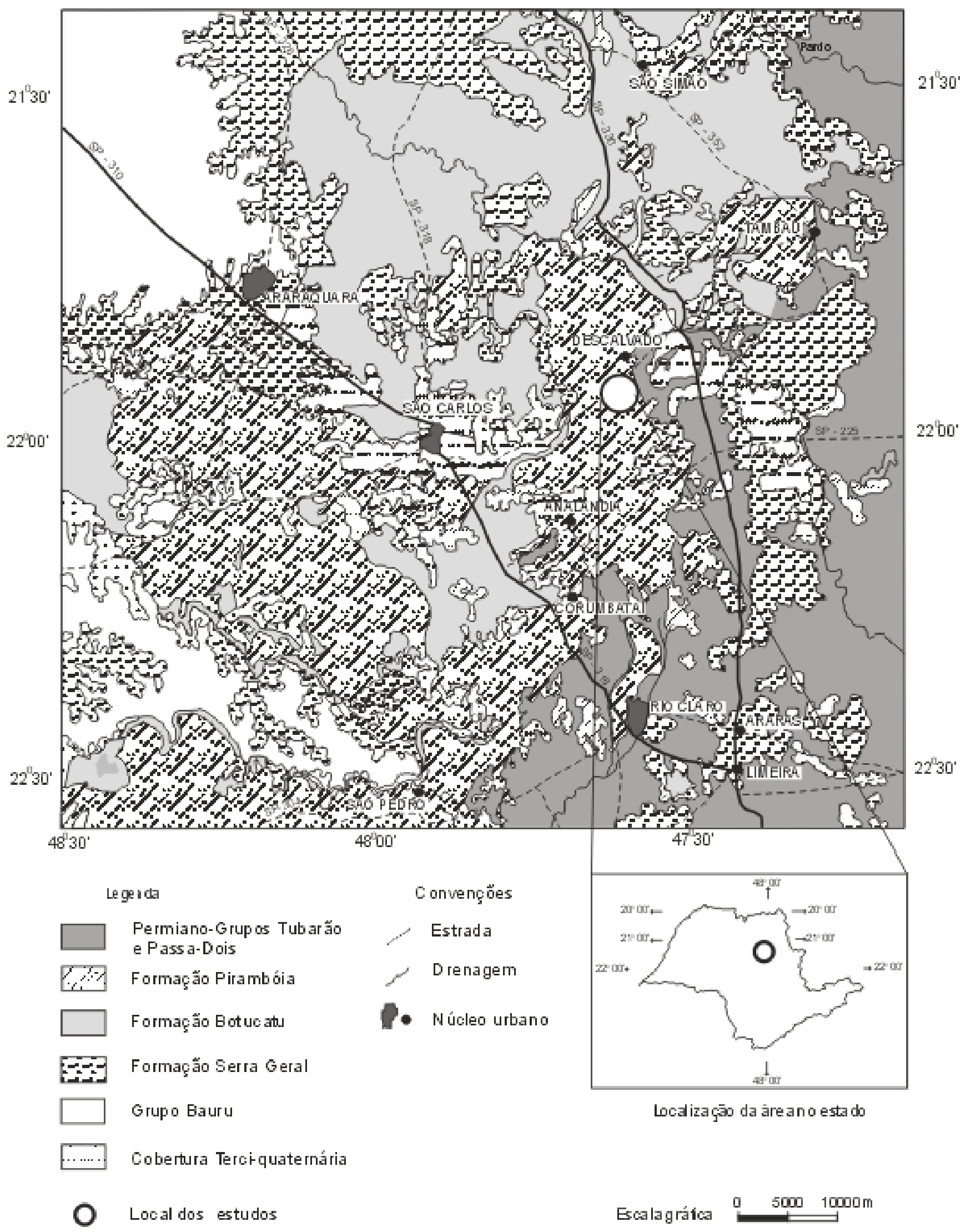

Figura 1 - Localização da área de estudos com relação à Bacia do Paraná (adaptado de Ferreira, 1995)

Figure 1 - Location of the study area in relation to the Paraná Basin (adapted from Ferreira, 1995) 
A lavra dos arenitos e dos sedimentos se dá a céu aberto em bancadas, que avançam pela encosta no sentido das cotas mais altas.

Parte dos arenitos situa-se abaixo do nível freático (NF) local original e, para que a lavra dos mesmos fosse possível, gradativamente o NF foi sendo rebaixado, causando a depressão deste junto à base do talude da bancada inferior.

Com este procedimento, o gradiente do lençol freático aumentou, aumentando também a velocidade de escoamento da água subterrânea, onde a mesma aflora na nova superfície do terreno.

Inicialmente a água aflora igualmente por toda a base do talude. Em seguida, em função da existência de pontos de maior permeabilidade, devido à granulometria do arenito ou à pequenos falhamentos, a água passa a aflorar preferencialmente por estes pontos de forma concentrada, originando nascentes. Estes fluxos de água passam a erodir o arenito que é friável, iniciando o processo de formação das cavidades (Fig. 2). Com a evolução deste processo as cavidades formadas podem aumentar bastante se transformando em cavernas, como é exemplificado pela foto 1 .

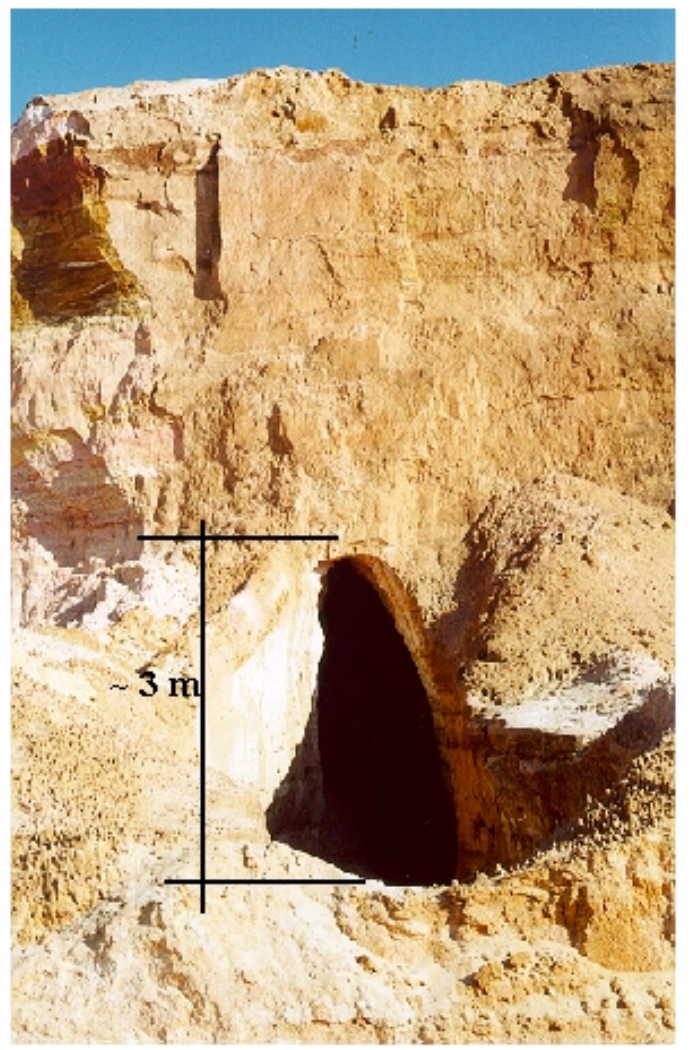

Foto 1 - Cavidade que ocorre na área, com aproximadamente $3 \mathrm{~m}$ de altura.

Photo 1 - Cavity in the area at a height of about $3 \mathrm{~m}$.
O tráfego dos veículos e máquinas utilizados na lavra dos arenitos, no piso superior desta bancada, pode provocar o abatimento do teto destas cavernas, implicando em sérios riscos. Desta forma, a identificação de técnicas adequadas à detecção prévia das cavidades existentes é muito importante para a continuidade e segurança da operação de lavra nestas áreas da jazida.

\section{ENSAIOS GEOFÍSICOS}

A escolha dos métodos gravimétrico, elétrico (resistividade) e GPR foi feita com base nos contrastes das propriedade físicas entre as cavidades e o arenito presente na área. A consulta a trabalhos anteriores como o de Hinze (1990) e de Ward (1990) também foi importantes na escolha dos dois primeiros métodos e o de Porsani (1999) para a escolha da técnica do GPR.

Os levantamentos geofísicos gravimétricos e de eletrorresistividade foram realizados em uma bancada onde, devido ao desmoronamento do teto, uma cavidade foi encontrada. Esta cavidade era alongada, com direção quase perpendicular à bancada, e no local do desmoronamento possuía uma altura de cerca de $5 \mathrm{~m}$ e $3 \mathrm{~m}$ de largura. Os ensaios com o GPR foram realizados após os levantamentos gravimétricos

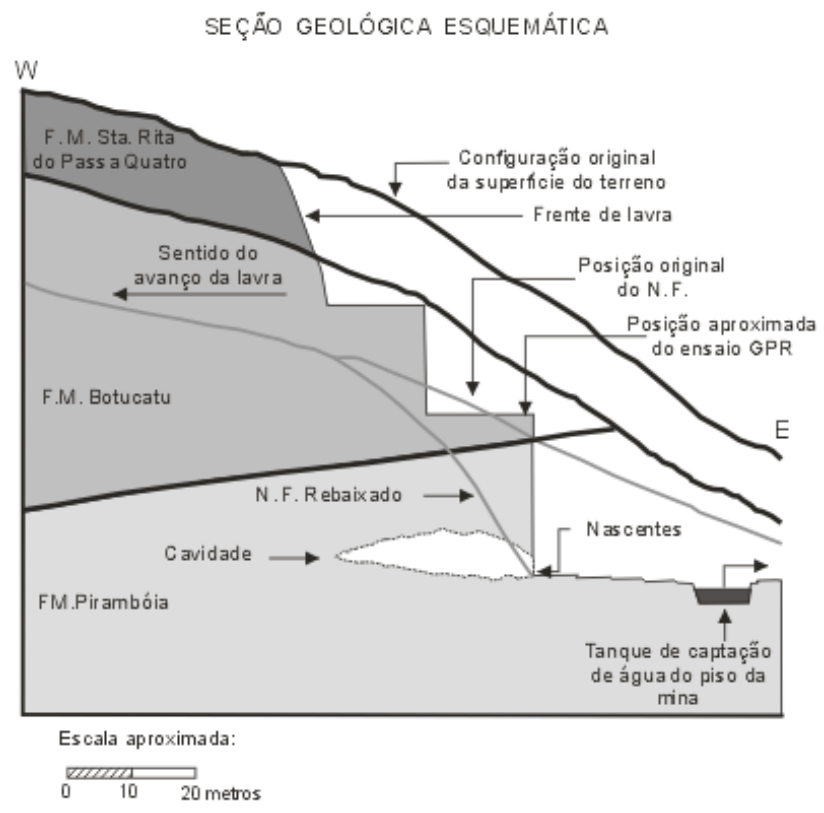

Figura 2 - Comparação entre a situação inicial e a situação atual da mina, mostrando o rebaixamento do nível freático e a conseqüente formação das cavidades.

Figure 2-Comparison between the initial situation and the current situation of the mine, showing the lowering of water table and the consequent formation of the cavities. 
e de resistividade, em uma bancada próxima, com linhas cortando uma cavidade existente.

$\mathrm{Na}$ bancada onde o estudo foi executado, a gravimetria e o caminhamento elétrico foram realizados em nove linhas paralelas, numeradas de L-1 a L-9, na direção NE e distanciadas 10 metros entre si e uma linha transversal denominada de LT-0. Estas linhas foram estaqueadas a cada $5 \mathrm{~m}$. A figura 3 mostra a localização da cavidade aflorante e das linhas ensaiadas.

Com o objetivo de cortar outras estruturas semelhantes àquela onde se encontra a cavidade, a linha L-4 foi estendida cerca de 260 m após o final das outras linhas.

\section{GRAVIMETRIA}

O levantamento gravimétrico foi realizado com um gravímetro LaCoste\&Romberg, modelo G, com precisão de 0,01 mGal. A metodologia de coleta de dados no campo foi desenvolvida para se executar um levantamento de detalhe, com leituras em um total de 269 estações, espaçadas entre de 5 metros ao longo das 9 linhas paralelas mostradas na figura 3 .

As medidas gravimétricas foram referenciadas à estação base localizada no Campus da UNESP na cidade de Rio Claro, cujo valor de g é: 978.587,24 mGal.

\section{Procedimento de Campo}

O levantamento gravimétrico foi conduzido de forma a cobrir a área de interesse com uma malha regular, disposta o mais simetricamente possível em torno do alvo de estudo. O espaçamento de $5 \mathrm{~m}$ entre as estações foi definido com base na modelagem mostrada na figura 4 , de maneira a se obter com bastante precisão as variações laterais das cavidades.

Esta modelagem inicial foi feita para verificar qual os valores de anomalia esperados considerando-se uma cavidade de forma trapezoidal, não aflorante, com o topo situado a $80 \mathrm{~cm}$ de profundidade, altura de aproximadamente $2 \mathrm{~m}$ e largura de $3 \mathrm{~m}$. Esta forma segue aproximadamente o padrão da cavidade presente na área. $\mathrm{O}$ contraste de densidade adotado foi o de $-1,6 \mathrm{~g} / \mathrm{cm}^{3}$. Este valor foi propositadamente conservador, visto que o arenito no local possui densidade em torno de $1,8 \mathrm{~g} / \mathrm{cm}^{3}$. O contraste entre a cavidade e o arenito deveria ficar próximo deste valor, no entanto, por segurança, considerou-se que próximo às cavidades o arenito poderia estar lixiviado, diminuindo o valor do contraste.

Este modelo apresentou uma anomalia em torno de 0,04 mGal, ou seja, quatro vezes maior que a precisão de leitura do gravímetro, e que o comprimento da metade da anomalia, ou seja, 0,02 $\mathrm{mGal}$ (o dobro da precisão de leitura) é da ordem de

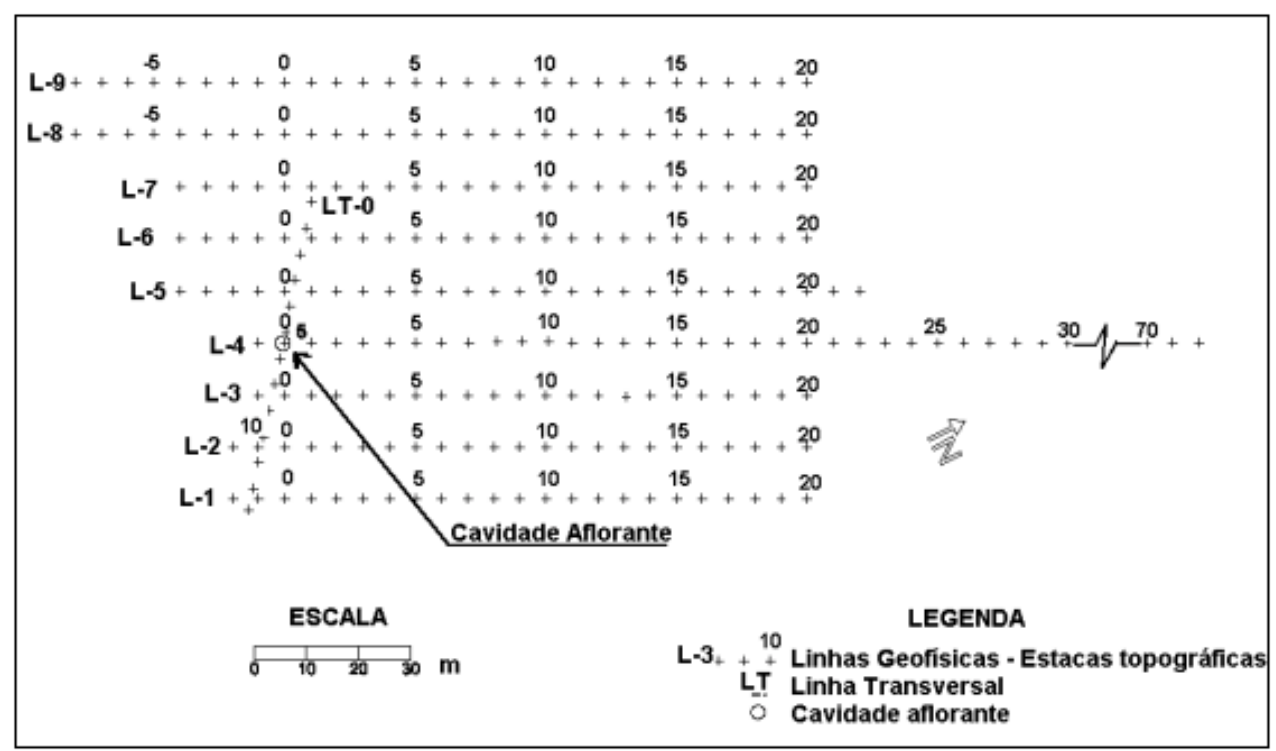

Figura 3 - Localização dos ensaios gravimétricos e elétricos. A cavidade aflora na superfície da bancada na estaca zero da Linha 4 e sua orientação segue aproximadamente a linha transversal LT-0..

Figure 3 - Location of the gravity and resistivity measurements. The cavity appears in the surface of the bench in the stake zero of the Line 4 and its orientation follows approximately the traverse line LT-0. 


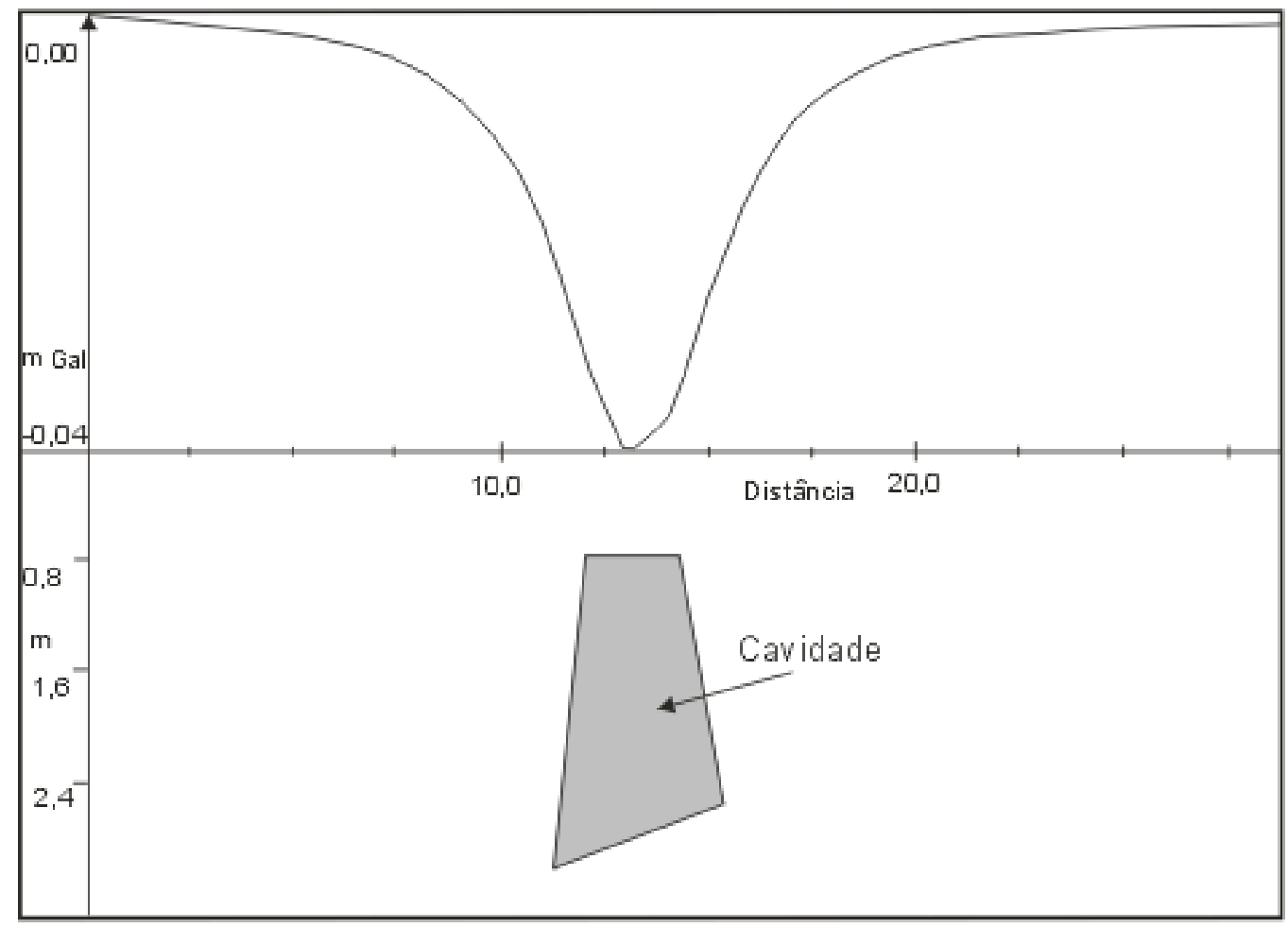

Figura 4- Curva de resposta obtida com o modelo de cavidade no arenito.

Figure 4 - Response curve obtained from the cavity model in the sandstone.

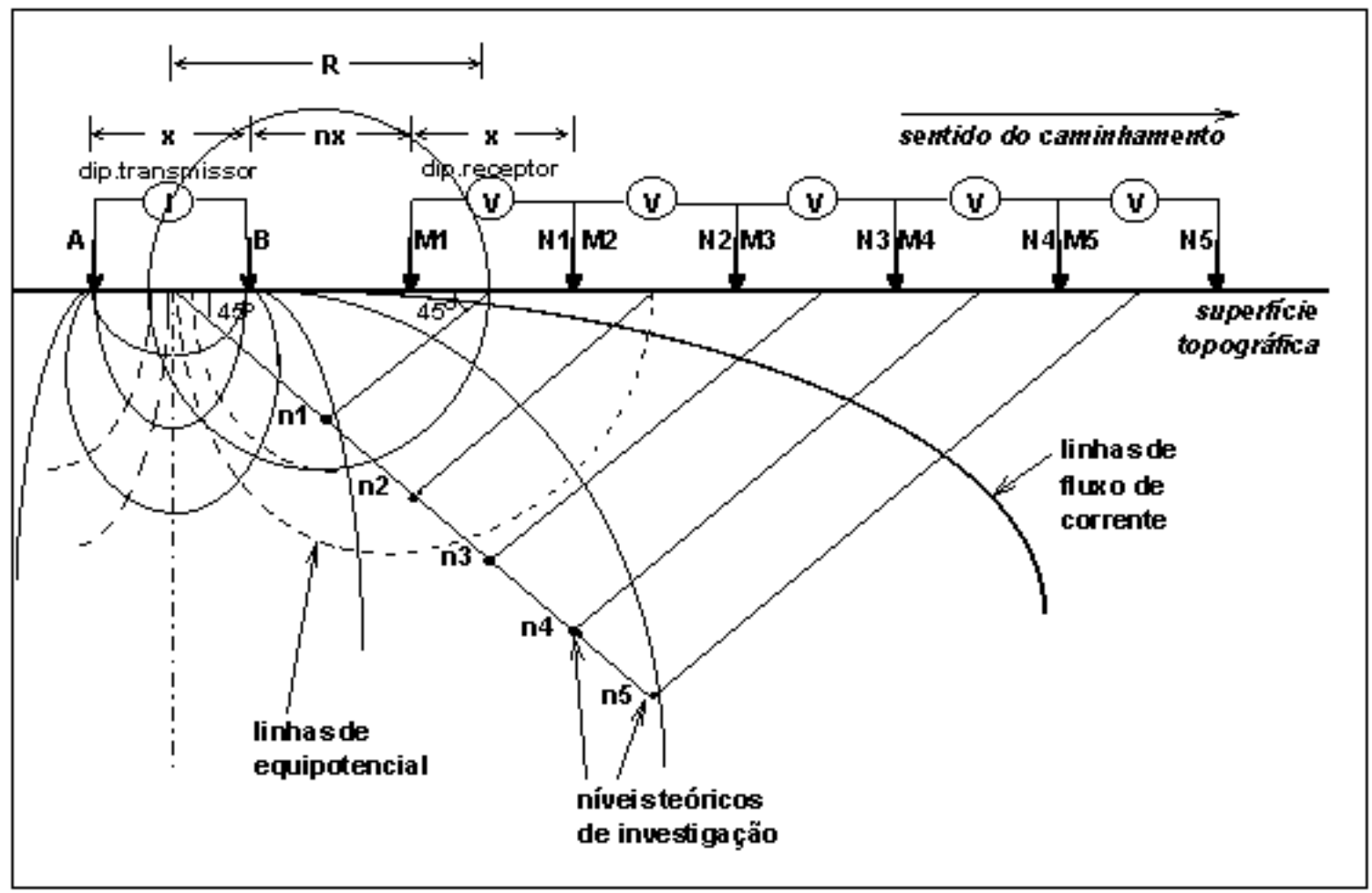

Figura 5 - Disposição no campo do arranjo Dipolo-Dipolo - técnica do caminhamento elétrico.

Figure 5 - Dipole-dipole electrical profiling - field array. 
$5 \mathrm{~m}$, definindo assim o espaçamento entre as estações de medida.

\section{Processamento dos dados}

As leituras gravimétricas obtidas durante o levantamento de campo foram processadas com o programa Gravpac da Lacoste-Romberg. Foram executadas as reduções necessárias para se obter os valores da anomalia Bouguer, que é formulada a partir do valor da gravidade observado no terreno, corrigido dos efeitos de maré, deriva instrumental e latitude, e do valor da gravidade normal calculado de acordo com esferóide de referência IGSN71 (1971 International Gravity Standardization Net) e reduzida ao nível da estação, de acordo com a fórmula:

$$
\Delta g_{B}=g_{\text {obs }}+0,3086 h-0,04191 \rho h+T \rho-g_{n}
$$

em que:

$\mathbf{g}_{\text {obs }}$ é o valor da gravidade observado

h é a elevação $(\mathrm{m})$

$\rho$ é a densidade $\left(\mathrm{g} / \mathrm{cm}^{3}\right)$

$\mathbf{g}_{\mathbf{n}}$ é o valor da gravidade normal

Como as anomalias a serem detectadas situamse muito próximas da superfície, optou-se por adotar um datum (h) também superficial, como uma maneira de ressaltar estas anomalias. Desta maneira o datum adotado foi a superfície do nível d'água, onde se espera que estejam as bases das cavidades presentes.

Portanto a densidade $\rho$ adotada para o cilindro utilizado na correção Bouguer foi $1,8 \mathrm{~g} / \mathrm{cm}^{3}$, que é a densidade do arenito.

\section{ELETRORRESISTIVIDADE}

Os valores de eletrorresistividade do local de ensaios foram levantados através da técnica de caminhamento elétrico efetuada ao longo da 9 linhas paralelas e na linha transversal LT-0 mostradas na figura 3. Devido ao caráter de detalhe e precisão dos resultados a serem obtidos, o arranjo utilizado no caminhamento elétrico $(\mathrm{CE})$ realizado no presente trabalho foi o Dipolo-Dipolo

O caminhamento elétrico foi executado utilizando-se espaçamento entre os dipolos de 5

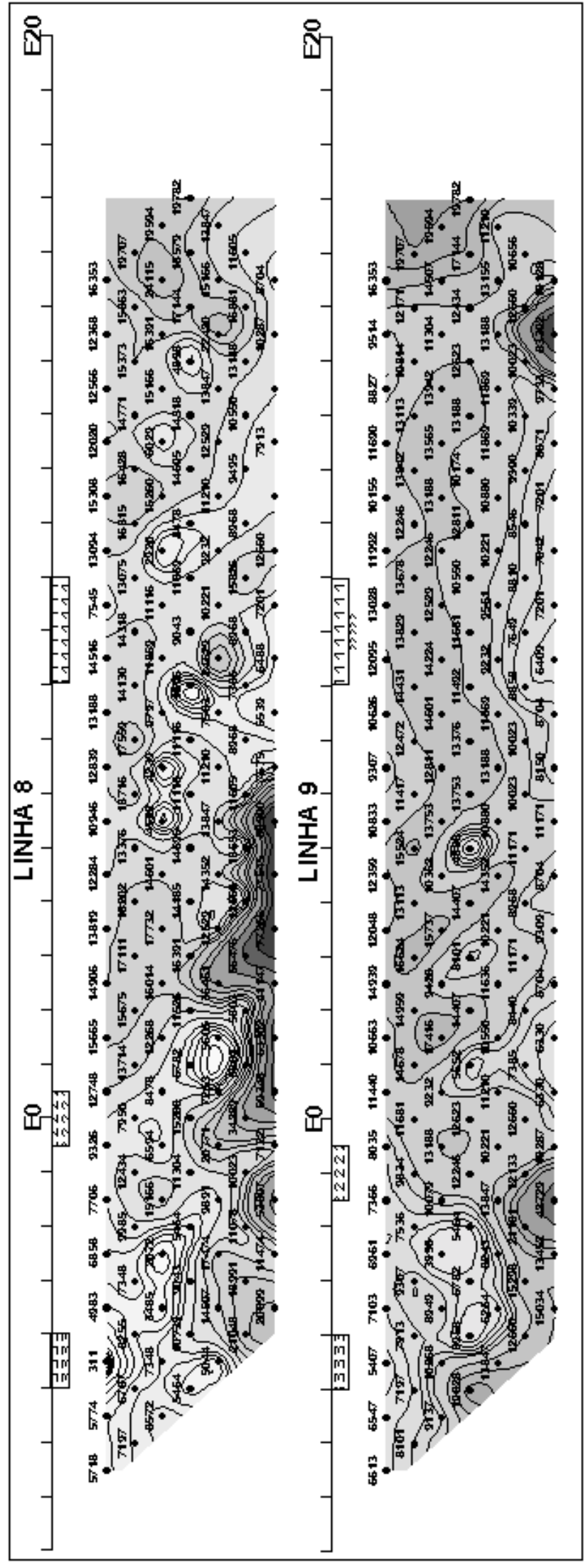

Figura 6 - Pseudo-seções de resistividade aparente das linhas 8 e 9. Figure 6-Apparent resistivity pseudo-sections of lines 8 and 9. 
m. O equipamento utilizado foi um resistivímetro digital da Bison Inc. modelo 2390.

\section{Procedimento de campo}

O ensaio foi desenvolvido ao longo de perfis previamente estaqueados, com espaçamento constante, em função das profundidades de investigações requeridas, pois tanto o espaçamento entre os dipolos como o número de dipolos utilizado regula as profundidades de investigações atingidas.

A figura 5 ilustra a disposição no campo, dos eletrodos de emissão de corrente e recepção de potencial, no desenvolvimento da técnica do CE arranjo de campo dipolo-dipolo.

Nesse tipo de arranjo a profundidade teórica atingida em cada nível investigado pode ser tomada como sendo $\mathrm{R} / 2$ (metros).

Como neste levantamento o espaçamento entre os dipolos foi de $5 \mathrm{~m}$ e tendo sido utilizados 7 dipolos, obteve-se sete níveis com profundidade teóricas de investigação, respectivamente, de: 5,0 - 7,5 - 10,0$12,5-15,0-17,5$ e $20,0 \mathrm{~m}$.

\section{Processamento dos dados}

O processamento dos dados de caminhamento elétrico foi iniciado com a elaboração das pseudosseções de resistividade aparente. Por meio destas seções foi possível identificar, através da projeção na superfície do terreno, as anomalias condutoras (em tons cinzas claros). Essas anomalias condutoras constituem-se, provavelmente, em faixas preferenciais por onde passa o fluxo d'água subterrâneo, tornandose por isso mais úmidas. A figura 6 mostra as pseudosseções obtidas para as linhas 8 e 9 da área.

Posteriormente, estes dados foram utilizados para se construir 3 mapas de isovalores de resistividade em três diferentes profundidades teóricas de investigação: 7,5 (sete e meio), 15 e 20 m. A execução destes mapas foi importante para se ter uma visão da distribuição tanto horizontal como vertical das anomalias determinadas.

\section{RADAR DE PENETRAÇÃO NO SOLO (GPR)}

Os ensaios com o radar de penetração foram feitos após os levantamentos gravimétricos e de resistividade elétrica, durante testes do equipamento do Laboratório de Geofísica Aplicada do Departamento de Geologia Aplicada do IGCE Unesp de Rio Claro-SP. O equipamento é o sistema RAMAC fabricado pela empresa Sueca MALA, Inc, com antenas bi-estáticas de 25, 50, 100200 e 400 $\mathrm{MHz}$.

\section{Procedimento de Campo}

O ensaio de campo foi feito sobre uma bancada próxima daquela onde se realizaram os ensaios gravimétricos e elétricos. Ele foi realizado sobre uma linha cortando uma cavidade que aflorava na base da bancada, de morfologia semelhante àquela mostrada na fotol.

\section{WARR}

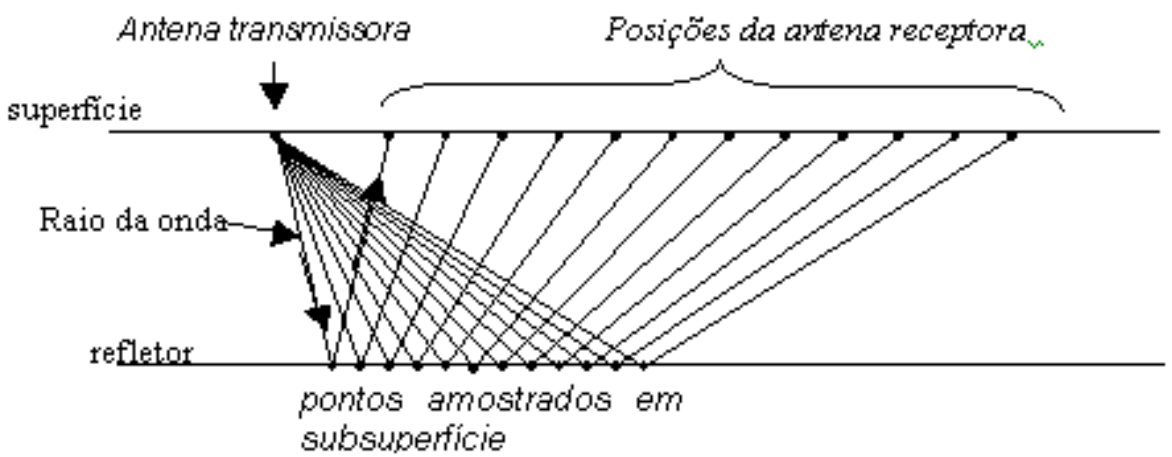

Figura 7 - Arranjo WARR realizado; as antenas utilizadas foram as de $25 \mathrm{MHz}$, sendo que a antena transmissora ficou fixa no início do arranjo e a antena receptora foi sendo deslocada com um incremento de $20 \mathrm{~cm}$.

Figure 7-WARR array carried out in this work. The used antennas were the $25 \mathrm{MHz}$. The transmitter antenna was fixed in the start of the array and the receiving antenna moved at $20 \mathrm{~cm}$ intervals. 


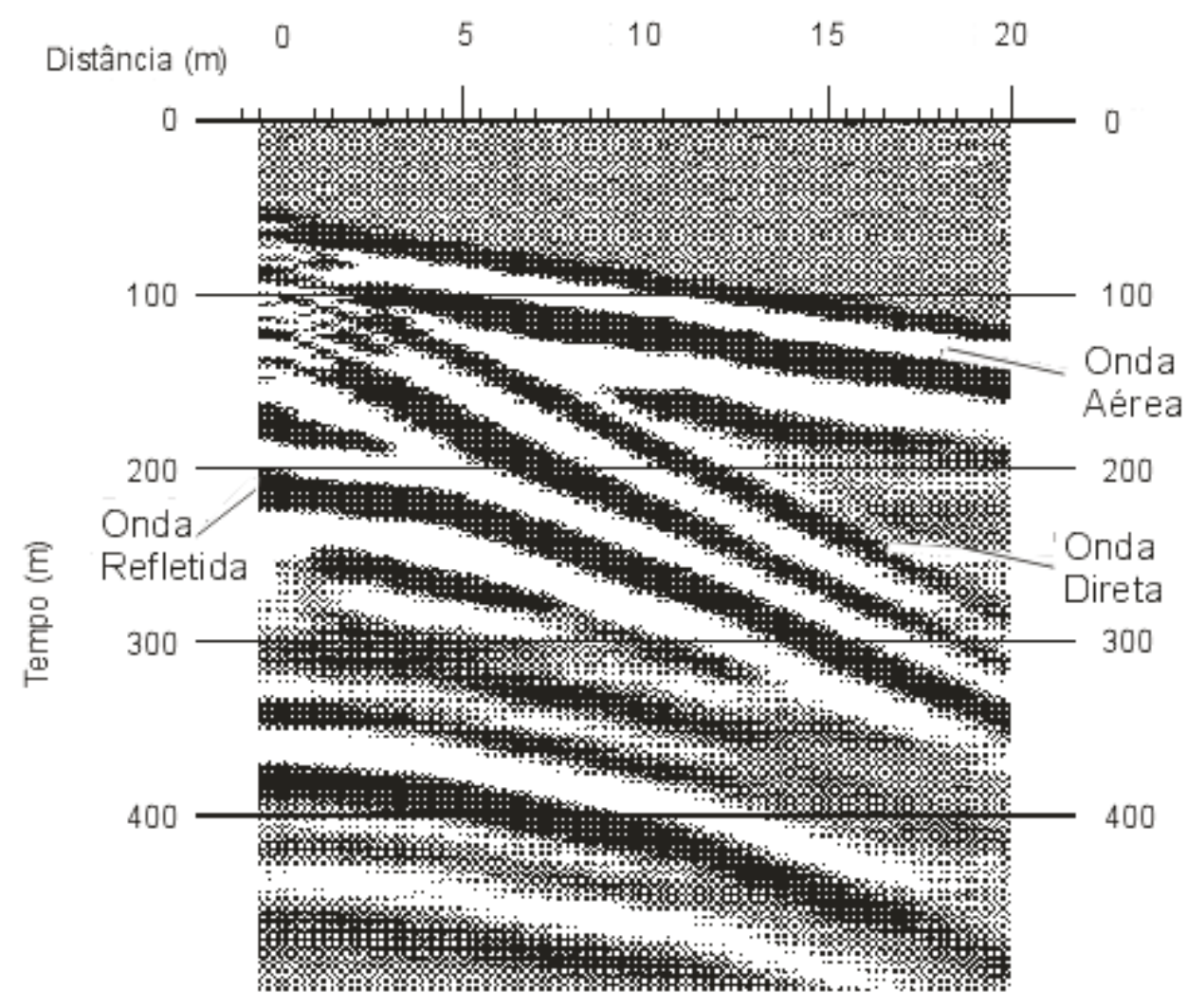

Figura 8 - Radargrama obtido com o arranjo WARR. É possível distinguir as retas correspondentes à chegadas da ondas aérea e direta e a hipérbole formada pela ondas refletidas.

Figure 8 - WARR array radargram obtained in this work. It is possible to distinguish the straight line corresponding to aerial and direct waves and the hyperbole formed by reflected waves.

\section{Common Offset}

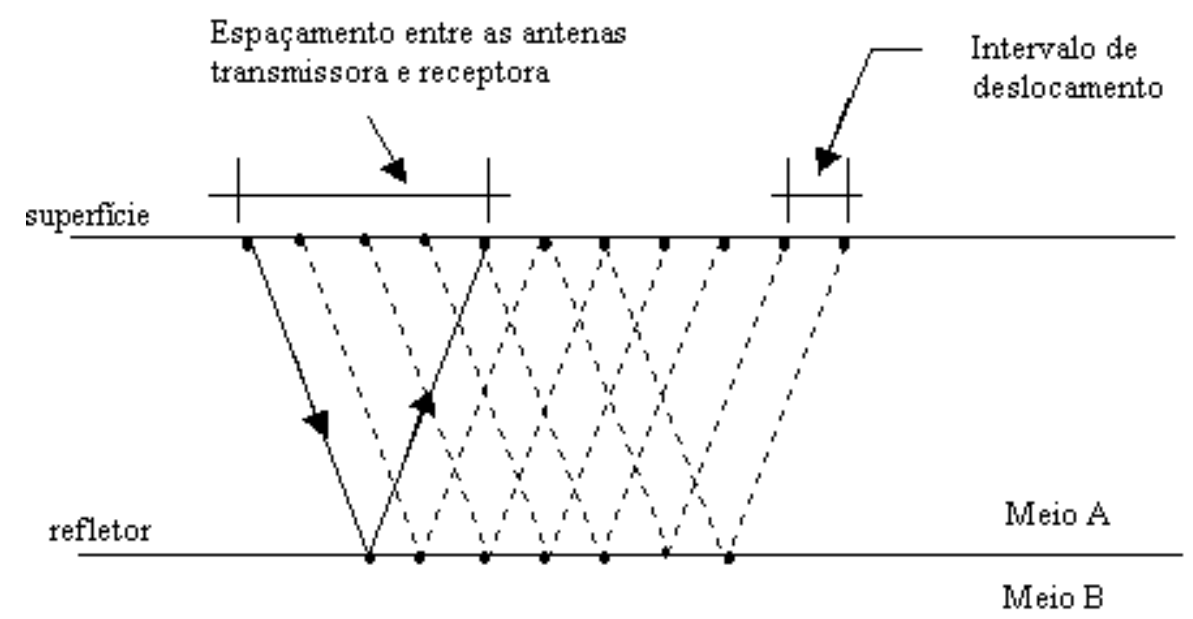

Figura 9 - Arranjo common offset, realizado, com as antenas de $100 \mathrm{MHz}$. O espaçamento entre antenas foi de $1 \mathrm{~m}$ e o intervalo de deslocamento de $20 \mathrm{~cm}$.

Figure 9-Common offset array carried out with $100 \mathrm{MHz}$ antennas. The spacing among antennas was $1 \mathrm{~m}$ and the displacement interval $20 \mathrm{~cm}$. 


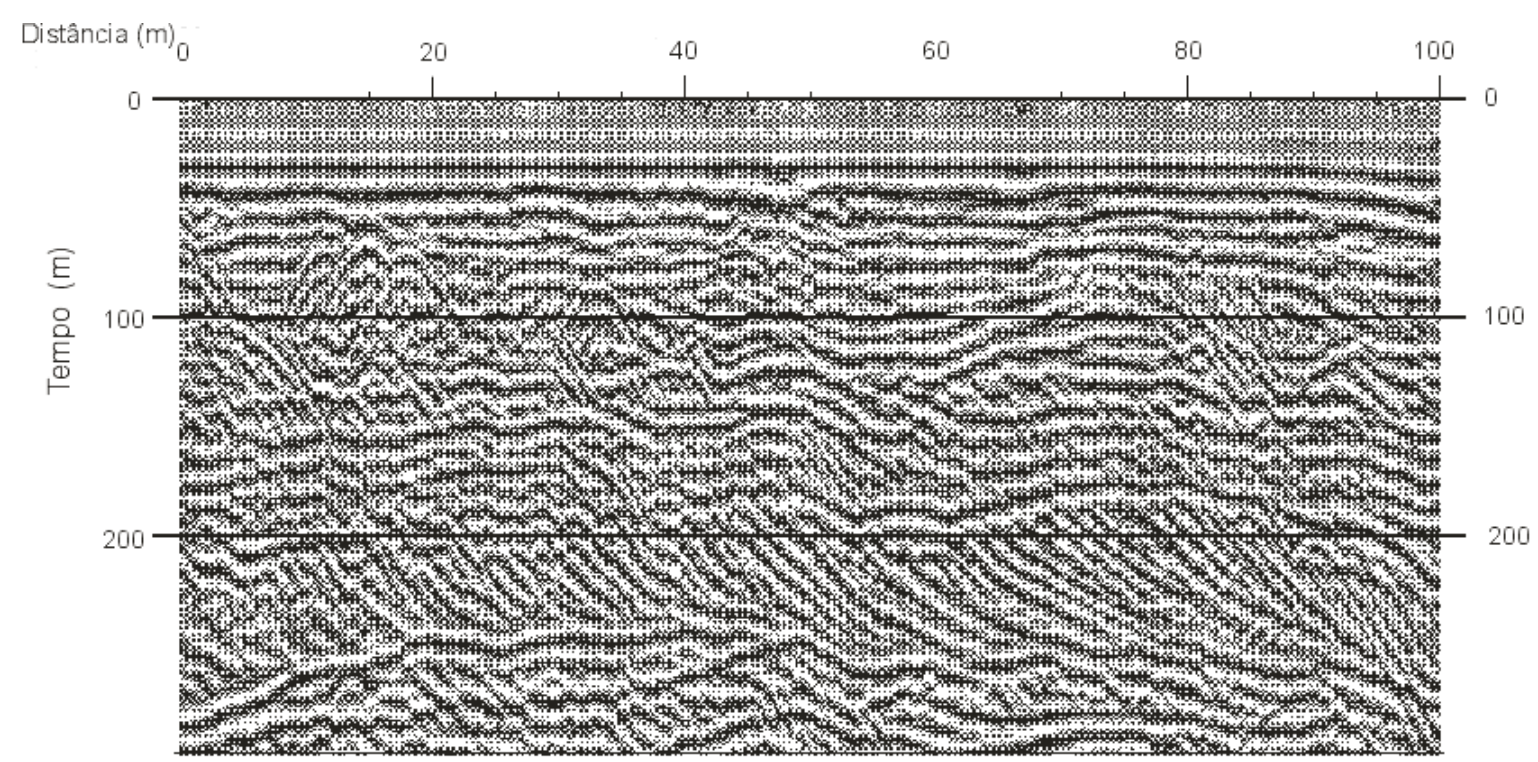

Figura 10 - Radargrama obtido do arranjo common offset, com antenas de $100 \mathrm{MHz}$. O offset foi de $1 \mathrm{~m}$, e o avanço de 20 em $20 \mathrm{~cm}$. Notar a presença de três hipérboles entre 10 e $20 \mathrm{~m}$, com ápices em torno de $60 \mathrm{~ns}$, interpretadas como geradas por cavidades no arenito.

Figure 10 - Radargram from common offset array using $100 \mathrm{MHz}$ antennas. The offset was of $1 \mathrm{~m}$, and the increment of $20 \mathrm{~cm}$. Note the presence of three hyperboles between 10 and $20 \mathrm{~m}$, with top at $60 \mathrm{~ns}$, interpreted as generated by cavities in the sandstone.

Foram realizados arranjos do tipo WARR (Wide Angle Reflection and Refraction) e common offset, como descritos por Annan (1992).

O arranjo WARR, cujo esquema de execução é mostrado na figura 7, teve o objetivo de determinar as velocidades de propagação das ondas eletromagnéticas. O radargrama obtido com este ensaio é mostrado na figura 8.

$\mathrm{O}$ arranjo common offset cujo esquema é mostrado na figura 9 , foi realizado em várias linhas. Um exemplo de seção common offset é mostrado na figura 10 .

\section{Processamento dos Dados}

O processamento dos dados de radar foi feito com o programa GRADIX, da Interpex Inc. e consistiu das seguintes etapas: edição dos radargramas, determinação das velocidades de propagação das ondas eletromagnéticas em subsuperfície e transformação do perfil GPR em profundidade.

A edição dos radargramas é um processo no qual procura-se melhorar a visualização das feições de interesse. Como no caso destes ensaios os dados de campo foram muito bons, devido a fatores como: bom contraste entre a anomalia (cavidades preenchidas com ar) e a encaixante (arenitos), alta resistividade elétrica dos arenitos e o baixo nível de ruídos da área, nesta etapa foram utilizados apenas a implementação do ganho dos traços do radargrama. $\mathrm{O}$ ganho que melhor evidenciou as anomalias de interesse, que no caso das cavidades pesquisadas formam feições hiperbólicas nos radargramas, foi o AGC (Automatic Gain Control), que foi aplicado na maioria dos radargramas em uma janela de $100 \mathrm{~ns}$ (nano segundo).

A determinação das velocidades de propagação das ondas eletromagnéticas no arenito baseou-se tanto no radargrama do arranjo WARR como nas hipérboles obtidas nos arranjos common offset. Nos locais onde ocorrem as cavidades, as velocidades obtidas variaram de 110 a $80 \mathrm{~m} / \mathrm{ms}$ (metros por microsegundos).

Para transformar as seções do radar de tempo em profundidade foram executados dois procedimentos. O primeiro correspondeu ao ajuste do tempo zero, utilizando a velocidade de propagação das ondas eletromagnéticas no ar $(\sim 300 \mathrm{~m} / \mathrm{ms})$ e determinando qual o tempo de propagação entre a antena transmissora e a receptora, e desta maneira fazendo a correção do tempo de chegada da onda aérea no radargrama. O segundo procedimento, que corresponde a transformação em profundidade do perfil GPR, foi feito utilizando-se a velocidade de propagação da onda eletromagnética no arenito. 


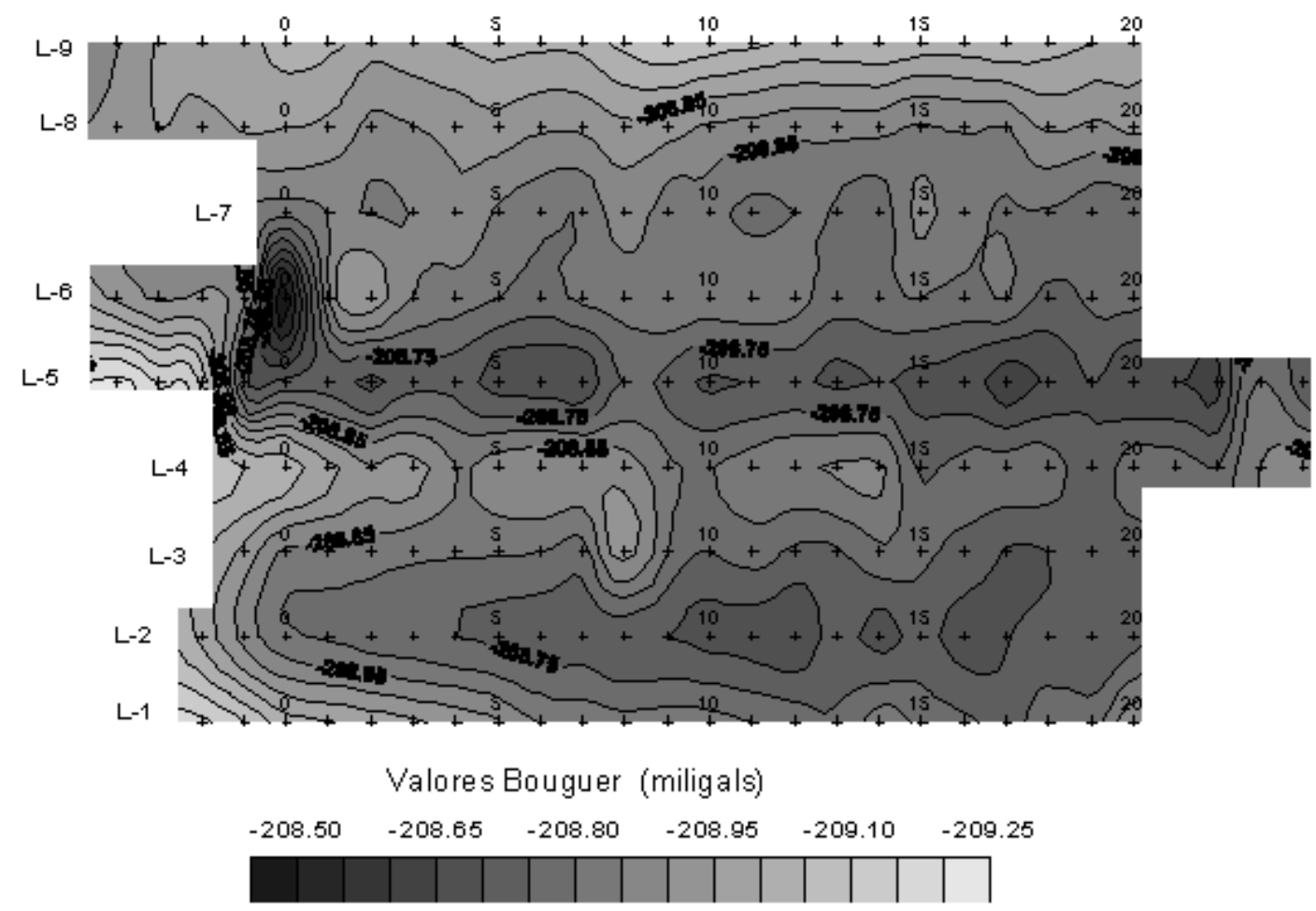

Figura 11 - Mapas de isovalores Bouguer para a área.

Figure 11 - Bouguer Map of the area.

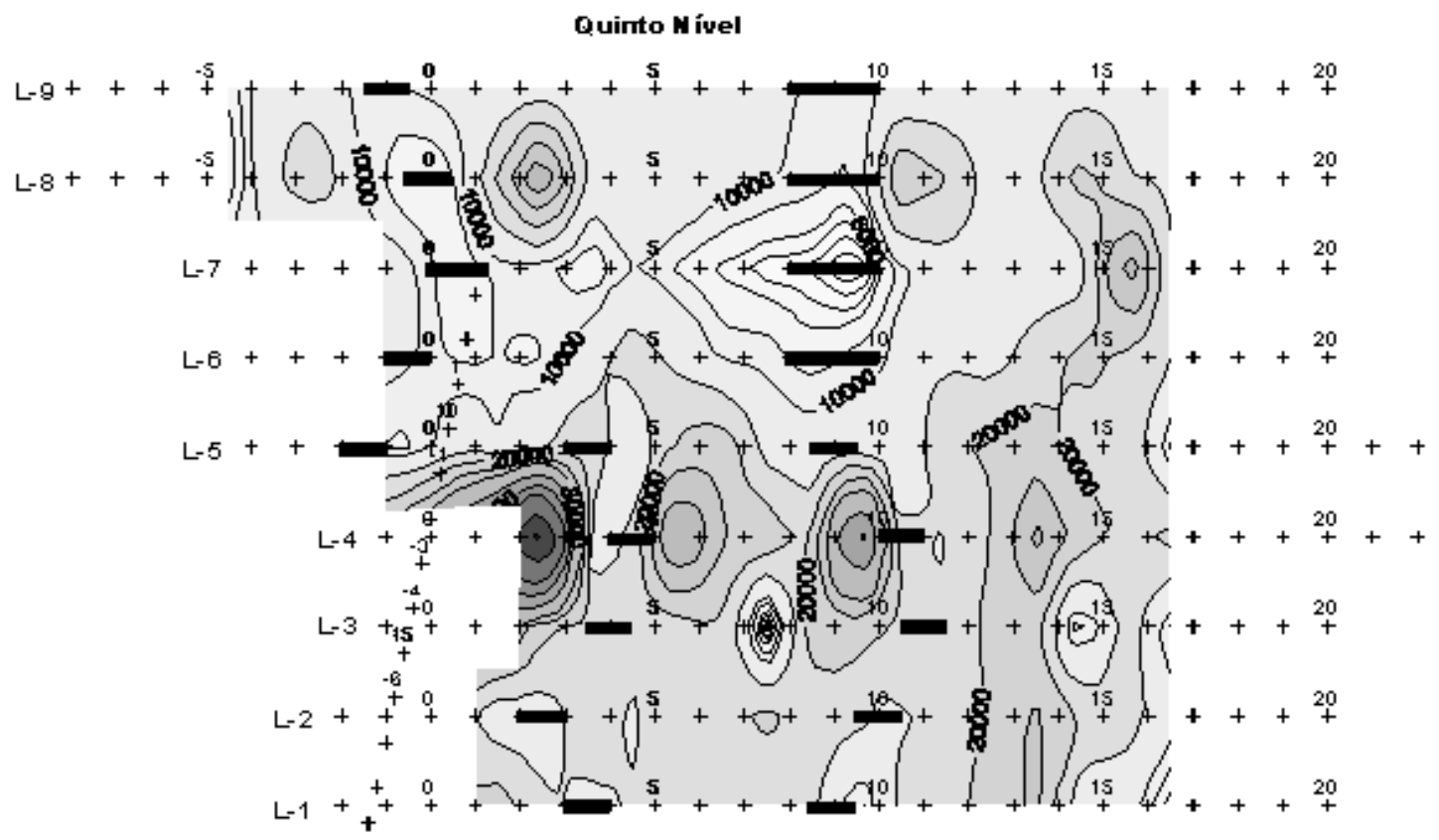

Resistividade (ohm.m)

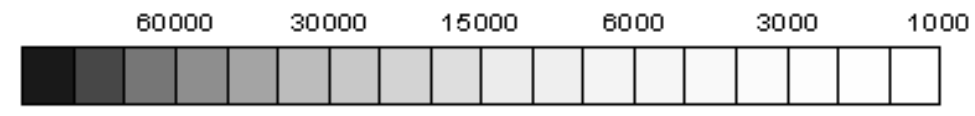

Figura 12 - Mapa de isovalores de resistividade para o quinto nível ensaiado, correspondente à profundidade teórica de investigação de $15 \mathrm{~m}$.

Figure 12 -Resistivity map of level five that corresponds to the theoretical depth of $15 \mathrm{~m}$ 


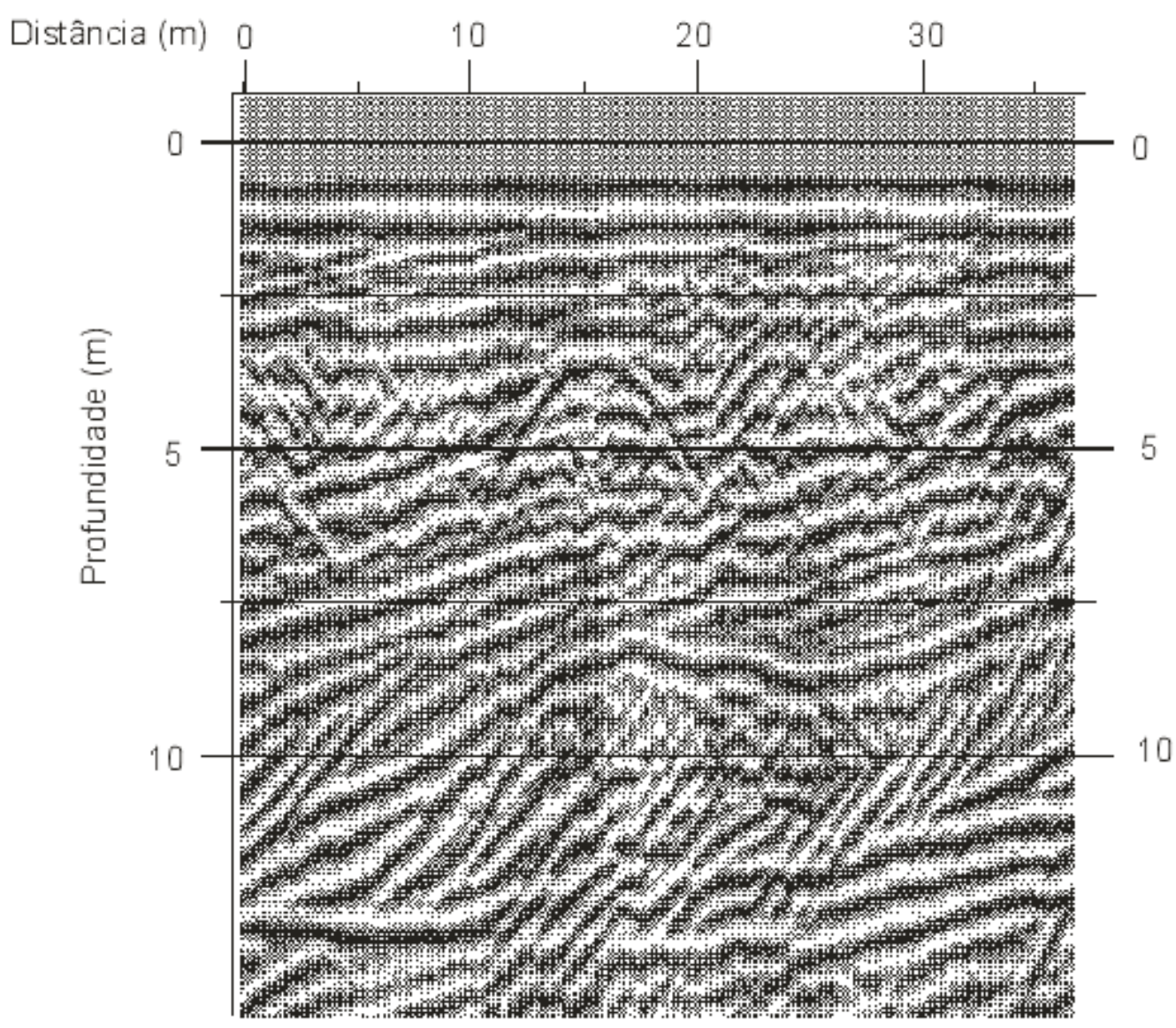

Figura 13 - Seção GPR em profundidade, mostrando uma feição hiperbólica bem definida, localizada em torno de $3 \mathrm{~m}$ de profundidade e a $15 \mathrm{~m}$ do início da linha. A velocidade de propagação das ondas eletromagnéticas que gerou esta hiperbole é de $102 \mathrm{~m} / \mathrm{ms}$, descartando portanto a possibilidade de ser ruído de fonte aérea. A velocidade utilizada para converter a seção de tempo para profundidade foi a determinada na hipérbole.

Figure 13-GPR depth section showing a well-defined hyperbolic feature located about $3 \mathrm{~m}$ of depth and from $15 \mathrm{~m}$ to the beginning of the line. The propagation velocity of electromagnetic waves defined by the hyperbole is $102 \mathrm{~m} / \mathrm{ms}$, which reject the possible aerial source noise. The velocity used to convert the time-depth section was determined by the hyperbole.

\section{RESULTADOS OBTIDOS}

Com os dados gravimétricos foi construído o Mapa dos Valores Bouguer determinados para área ensaiada. Neste mapa, mostrado na figura 11, os baixos gravimétricos estão em cinza claro e os altos gravimétricos estão em cinza escuro.

Com dos dados de resistividade obtidos com o caminhamento elétrico foram inicialmente construídas as pseudo-seções ao longo dos perfis ensaiados. Destes perfis foram extraídos mapas com curvas de isovalores de resistividades para várias profundidades teóricas de investigação. Na figura 12 é mostrado o mapa referente ao $5^{\circ}$ nível de investigação, equivalente à profundidade teórica de $15 \mathrm{~m}$. Neste mapa as altas resistividades estão representadas pelos tons cinza escuros e as baixas resistividades pelos tons cinzas claros.
Com os ensaios GPR foram levantadas várias seções que cortam perpendicularmente o eixo maior de cavidades conhecidas. $\mathrm{O}$ radargrama mostrado na figura 13 é um exemplo da feição hiperbólica que as cavidades formam nos radargramas.

\section{DISCUSSÃO DOS RESULTADOS}

Analisando-se os mapas de valores Bouguer e de isovalores de resistividade verifica-se que, no local da cavidade aflorante, existe uma correlação bastante boa entre as anomalias esperadas e as obtidas. Como pode ser constatado no mapa de valores Bouguer, a ocorrência de um baixo gravimétrico nesta região é diretamente correlacionável com a presença de um corpo menos denso. Com relação aos valores de resistividade, eles também indicam a presença da cavidade através dos altos valores de resistividade. 
Outra constatação que pode ser feita, analisandose os dois mapas conjuntamente, é a coincidência entre a localização de anomalias de alta resistividade com anomalias de baixos valores Bouguer. Estas áreas estão provavelmente lixiviadas pelo fluxo da água subterrânea, ao longo de direções preferenciais.

No local da anomalia presente na linha 8 , nas proximidades da estaca 3, no mapa de resistividade (figura 12), foi encontrada uma cavidade, após o avanço da bancada.

Quanto aos ensaios de GPR, pode-se notar nos radargramas apresentados, não só as excelentes feições hiperbólicas que são relacionadas às cavidades, mas também estruturas estratigráficas bem definidas.

\section{CONCLUSÃO}

Os resultados obtidos no presente trabalho mostram que a gravimetria, e a eletrorresistividade, com a técnica do caminhamento elétrico, combinados com o GPR apresentaram bons resultados na detecção de cavidades superficiais em arenitos, incentivando o seu emprego integrado em trabalhos desta natureza.

Com relação ao GPR, deve-se ressaltar que a boa qualidade obtida nos arenitos pode não se repetir em rochas de menor resistividade elétrica, pois nestes casos a atenuação que ondas eletromagnéticas vão sofrer pode comprometer a qualidade dos radargramas.

\section{REFERÊNCIAS}

Annan, A. P., 1992. Uses and techniques of ground penetrating radar in near-surface geophysics.
Workshop Notes: Society of Exploration Geophysicists, Sensors \& Software Inc., 110 pp.

Ferreira, G.C., 1995. Estudo dos mercados produtor e consumidor de areia industrial no Estado de São Paulo. Tese de Doutorado. Instituto de Geociências e Ciências Exatas, Unesp, 142 pp.

Hinze, J. W., 1990. The role of gravity and magnetic methods in engineering and environmental studies. Geotechnical and Environmental Geophysics. Series ins Geophysics N. 5. Ed. Stanley H. Ward - Tulsa - Society of Exploration Geophysicists Vol I-Review and tutorial - pp 75-126.

Massoli, M., 1981. Geologia do município de $\mathrm{St}^{\mathrm{a}}$ Rita do Passa Quatro, SP - Revista do Instituto Geológico.

Petri, S., Fulfaro, V. J., 1983. Geologia do Brasil (Fanerozóico) - São Paulo: T. A. Queiroz - Ed. da Universidade de São Paulo.

Porsani, J. L., 1999 Ground penetrating radar (GPR): proposta metodológica de emprego em estudos geológico-geotécnicos nas regiões de Rio Claro e Descalvado - SP. Tese de Doutorado. Instituto de Geociências e Ciências Exatas, Unesp, $145 \mathrm{pp}$.

Soares, P. C., 1973. O Mesozóico Gonduânico no Centro-leste do Estado de São Paulo - Tese de Doutorado. Faculdade de Filosofia Ciências e Letras de Rio Claro.

Ward, S. H., 1990. Resistivity and Induced Polarization Methods. Geotechnical and Environmental Geophysics. Series in Geophysics N. 5. Ed. Stanley H. Ward - Tulsa - Society of Exploration Geophysicists - Vol I - Review and tutorial. - pp 147-185.

\section{DETECTION OF CAVITIES IN SANDSTONE USING GRAVITY, RESISTIVITY AND GPR METHODS}

Locating, or even the simple detection of subterranean cavities has always posed a challenge using the available geophysical and prospecting techniques. Examining the multiple morphological facies that can be generated by cavities, either of natural origin (karst, erosive features, etc.) or those created our caused by human activity (tunnels, mines, etc.), there is still no "recipe" for determining which geophysical techniques to utilize for a given set of circumstances or problems.

This article shows the results of a geophysical study that was carried out around a sand pit/quarry known to contain subterranean cavities, near Descalvado, São Paulo. The geologic units found in this study area are as follows: the Pirambóia Formation (Triassic-Jurassic), the Botucatu Formation (Jurassic-Cretaceous), diabase intrusions (Cretaceous), and the Santa Rita do Passa Quatro Formation (Tertiary). The principal objective of the study was the detection of cavities within the sandstones or sandy facies of the Pirambóia e Botucatu formations. These cavities were indirectly caused by human activity, i.e., the lowering of the water table for the purpose of sand extraction. The geophysical techniques utilized were as follows: gravity method, resistivity (dipole-dipole electrical profiling) and Ground Penetrating Radar (GPR). The choice of these 
methods was done based on the contrasting physical properties of the cavities and the sand. Review of previous research of this type, such as that done by Hinze (1990) and Ward (1990), also influenced our choice of the first two methods, whereas work done by Porsani (1999) influenced the choice of the GPR technique.

The gravity and dipole-dipole measurements were taken on an embankment where, due to the collapse of the roof a cavity cropped out at the surface. This cavity, measuring $5 \mathrm{~m}$ in height and $3 \mathrm{~m}$ in width, was elongated in a direction almost perpendicular to the embankment.

The readings with the GPR were made done following gravity and resistivity measurements on an adjacent embankment, with lines crossing the existing cavity.

For gravity measurements a model G Lacoste\&Romberg gravimeter, with $0.01 \mathrm{mGal}$ precision, was employed. The data collection method was carried out so that detailed coverage was obtained. Readings were taken at 269 stations; 5-meter intervals along 9 parallel lines (Figure 3). The 5-meter spacing was determined based on modeling shown in Figure 4 . The gravity measurements were referenced to a base station located at UNESP - Rio Claro, whose g value is 978,587.24 mGal. The processing of these data was carried out using the program Gravpac provided by Lacoste\&Romberg.

The resistivity values in the study local were obtained through the electrical profiling technique, in dipole-dipole configuration, using 5-meter dipole spacing, along both the 9 parallel lines and a transverse line (LT-0) shown in Figure 3. Measurements were taken using a digital 2390 Bison Inc. resistivity meter.

The results obtained with these two techniques that they are complimentary under these conditions. The gravimetry shows an "absence of mass" adjacent to the surface cavity and the dipole-dipole readings display high electrical resistivity values in the same area.

The GPR survey employed the WARR (Wide Angle Reflection and Refraction) array and the common offset described by Annan (1992). The WARR array was used to determine the propagation velocities of the electromagnetic waves. Processing of the raw radar data, done with the GRADIX program (created by Interpex, Inc.), involved the following steps: editing of radargrams, determination of the propagation velocities in the subsurface and the transformation of the GPR profile with depth. This technique detected the presence of cavities by means of the occurrence of hyperbolic facies that appeared on the radargrams.

The results obtained by this study show that gravimetry, and electroresistivity (the electrical caminhado technique), combined with GPR yield good results in the detection of surface cavities in sandy soils and sandstones. As such, these positive results are an incentive to employ these methods in future investigations and work of this nature.

\section{NOTE ABOUT THE AUTHORS}

\section{João Carlos Dourado}

Bacharel em Geologia pelo Instituto de Geociências da Universidade de São Paulo (USP) em 1977. Doutor em Geociências pela Unesp em 1997. Trabalhou com geofísica aplicada no Instituto de Pesquisas Tecnológicas do Estado de São Paulo de 1977 a 1997. Atualmente é professor assistente doutor do Departamento de Geologia Aplicada do IGCEUnesp onde atua desde 1997. Trabalha com a aplicação de métodos geofísicos à Hidrogeologia, Meio Ambiente, Geologia de Engenharia e Geotectônica. E-mail:jdourado@rc.unesp.br

\section{Walter Malagutti Filho}

Bacharel em Geologia pelo Instituto de Geociências e Ciências Exatas (IGCE) da Universidade Estadual Paulista (Unesp) em 1980. Doutor em Geociências pela Unesp em 1991. Trabalhou com geofísica aplicada no Instituto de Pesquisas Tecnológicas do Estado de São Paulo de 1981 a 1986. Atualmente é professor assistente doutor do Departamento de Geologia Aplicada do IGCE-Unesp onde atua desde 1986. Trabalha com a aplicação de métodos geofísicos à
Hidrogeologia, Meio Ambiente, Geologia de Engenharia e Geotectônica.E-mail:malaguti@rc.unesp.br

\section{Antônio Celso de Oliveira Braga}

Bacharel em Geologia pelo Instituto de Geociências e Ciências Exatas (IGCE) da Universidade Estadual Paulista (Unesp) em 1978. Doutor em Geociências pela Unesp em 1997. Trabalhou com geofísica aplicada no Instituto de Pesquisas Tecnológicas do Estado de São Paulo 1978 a 1995. Atualmente é professor assistente doutor do Departamento de Geologia Aplicada do IGCE-Unesp. Trabalha com a aplicação de métodos geofísicos à Hidrogeologia, Meio Ambiente e Geologia de Engenharia. E-mail: acobraga@rc.unesp.br

\section{Noedir Nava}

Bacharel em Geologia pela Faculdade de Filosofia e Ciências e Letras de Rio Claro, SP em 1974. Exerce desde 1975 a função de Gerente de Geologia da Mineração Jundu, em Descalvado. 